\title{
Messages from hypothesis-driven genotyping: the case of schizoaffective disorder, bipolar type
}

\author{
Molecular Psychiatry (2010) 15, 113-114; \\ doi:10.1038/mp.2009.153
}

There are currently two main approaches to genotyping: hypothesis-free genome-wide association studies (GWAS), in which a very large number of singlenucleotide polymorphisms (SNPs) in the range of several hundred thousand to over a million are associated with cases versus controls, ${ }^{1-3}$ or a hypothesis-based approach, in which SNPs along relevant, hypothesis-driven pathways are examined. ${ }^{4,5}$ In this issue of Molecular Psychiatry, Craddock et al. ${ }^{6}$ show that these approaches are not as dichotomous as they may seem: the well-known psychiatric genetics group from Cardiff, Wales and collaborators used a promising result from previous GWAS analysis that pointed to a gamma-aminobutyric acid $(\mathrm{GABA})_{\mathrm{A}}$ receptor $\beta 1$ subunit, GABRB1. They used this hypothesis-free GWAS finding as a starting point for a hypothesisdriven approach aimed at examining variants in genes encoding various GABA receptor subunits in several bipolar phenotypes.

The strongest association was with GABRB1 SNP rs7680321 and the Research Diagnostic Criteria (RDC) phenotype of schizoaffective disorder, bipolar type (SABP). This describes individuals who, in addition to clear-cut episodes of mania, show psychotic symptoms (delusions and/or hallucinations) that are not easily understood as being the result of extreme mood change and that are often seen also in individuals diagnosed with schizophrenia. It does not include all the people with bipolar disorder with psychosis. $^{7}$ When those meeting the RDC criteria for schizoaffective disorder are removed from the bipolar sample, the statistical significance of the association is lost. This raises the issue of heterogeneity of large diagnostic categories in psychiatry and suggests that distinct phenotypic subgroups may have distinct genetic backgrounds. I feel confident to categorically state that no single diagnostic group in psychiatry will have a specific genetic marker that is present in all individuals with the disorder, as currently diagnosed by ICD-10 or DSM-V. Our disease categories are clinical constructs of people who present with similar clinical features that may be caused by the most varied combinations of small genetic effects, modified by epigenetics, as well as pre- and post-natal environmental experiences. What we study is not a linear outcome of gene variation causing alterations in protein, and leading to specific changes in function, with disease as the ultimate and predictable outcome of such changes in genetic sequence. This paper clearly illustrates that.

Moreover, the authors found associations with noncoding areas. Whether the genetic variants they identified as significantly associated with SABP are of a regulatory nature remain to be determined. Moreover, these are present only in that specific subtype of bipolar disorder, SABP. Although in recent times there has been a push to only accept genetic findings from very large samples, the authors show here a high level of significance, with only 279 patients who met the RDC criteria for SABP. This may represent a shifting of the pendulum from very large studies of mixed cases to smaller studies of welldefined phenotypes.

One can scrutinize these findings from a very specific level or take a broader view. Is this a half empty or half full glass? What is the meaning of a noncoding association with a specific clinical subtype of small $n$ ? A very positive outcome of this study is that going from a hypothesis-free starting point, in which various bipolar subtypes were tested, they were able to identify something specific for SABP that is not shared by schizophrenia. Schizoaffective disorder has been much debated as a clinical subtype and its differentiation from schizophrenia has been questioned. Although several papers point out to a continuum between bipolar and schizophrenia with common genetic variants associated with both disorders, Craddock et al. report in this issue of Molecular Psychiatry that the genetic association they uncovered was for the subtype of bipolar disorder most closely connected with schizophrenia, namely schizoaffective disorder; it was remarkably specific for bipolar and could not be reproduced in patients with schizophrenia: The authors found no evidence of association when, using the same methodology and genotyping platform, they examined the set of SNPs at these (GABA) genes within their sample of 476 white UK cases meeting the DSMIV criteria for schizophrenia.

A logical conclusion is that small effect genes that cause psychotic disorders are distributed in different patterns. Some variants may be common across the full bipolar-schizophrenia spectrum, whereas others may be specific to distinct clinical phenotypes. It is therefore plausible that the combination of genetic susceptibility factors that are not specific for a distinct psychiatric subphenotype with those that are may underlie specific presentations such as schizoaffective disorder. 
Craddock et al. report here that they 'do not believe that 'schizoaffective disorder' in general, or RDC schizoaffective disorder in particular, is a neatly defined, discrete, biological diagnostic entity.' But then what is? Particularly in psychiatry, it is unimaginable to me that there will be any time soon 'neatly defined, discrete, biological diagnostic entities.' Two key features of the fundamental biological systems underlying psychiatric disorders preclude that: pleiotropy and redundancy. The systems of interest in psychiatry, including GABA, are very pleiotropic. In other words, the same system can cause multiple biological effects and may therefore be implicated in various disorders. GABA, for example, has been implicated not only in SABP but also in alcohol abuse, anxiety states and panic episodes. Redundancy describes the fact that the same function can be controlled by multiple systems. This is of great evolutionary importance as it ensures that disruption of one system will not cause major alterations of key vital functions. The challenge for our field is to parcel out multiple and small contributions of pleiotropic and redundant systems to complex and highly heterogeneous phenotypes that are the common final outcome of a very high number of possible combinations of interactions of myriad genetic and environmental factors.

\section{J Licinio \\ John Curtin School of Medical Research, The Australian National University, Canberra, ACT, Australia E-mail: julio.licinio@anu.edu.au}

\section{References}

1 Muglia $\mathrm{P}$ et al. Mol Psychiatry (advance online publication 23 December 2008); doi: 10.1038/mp.2008.131.

2 Psychiatric GWAS Consortium Steering Committee. Mol Psychiatry 2009; 14: 10-17.

3 Mitchell KJ, Porteous DJ. Mol Psychiatry 2009; 14: 740-741.

4 Wong ML et al. Proc Natl Acad Sci USA 2006; 103: 15124-15129.

5 Wong ML et al. Mol Psychiatry 2008; 13: 800-812.

6 Craddock N et al. Mol Psychiatry 2010; 15: 146-153 (this issue).

7 Hamshere ML. Br J Psychiatry 2009; 195: 23-29. 